\title{
HANASUI: Multi-view Observable and Movable Fogscreen
}

\author{
Yu Ishikawa ${ }^{1}$, Masafumi Muta ${ }^{1}$, Junki Tamaru ${ }^{1}$, Eisuke Nakata ${ }^{2}$, \\ Akira Uehara $^{2}$, and Junichi Hoshino ${ }^{3}$ \\ ${ }^{1}$ University of Tsukuba, Graduate School of System and Information, Tsukuba, Japan \\ \{ishikawa.yu, mecab\} @entcomp.esys.tsukuba.ac.jp, \\ tamaruafz.iit.tsukuba.ac.jp \\ ${ }^{2}$ University of Tsukuba, School of Engineering, Tsukuba, Japan \\ \{s1011195, s1111088\}@u.t.sukuba.ac.jp \\ ${ }^{3}$ University of Tsukuba, Faculty of Engineering, Information and Systems, Tsukuba, Japan \\ jhoshino@esys.tsukuba.ac.jp
}

\begin{abstract}
In this paper, we propose the method for creating multi-view movable fogscreen, and then implement it in our system called "HANASUI". "HANASUI" displays handheld-like fireworks through a fog screen instead of sparkles. Our method generates projection data dynamically from a virtual space and then casts it with multiple projectors, tracking the marker attached to the device which spouts fog at the fogscreen with infrared cameras and infrared floodlights. Finally, we conducted a survey to verify the capabilities of "HANASUI" and its potential for art and entertainment purposes.
\end{abstract}

Keywords: Fogscreen, Multi-View Observable, Projection Mapping, Entertainment.

\section{$1 \quad$ Introduction}

A fogscreen, which uses fog as a projector screen, has proposed as a technological system for event staging or information presentation [1,2]. A feature of the fogscreen is that, unlike conventional displays that have used liquid crystal, it doesn't have a clearly defined edge or the edge is subtle. Another feature is that, because the screen is made of fog, the projected image can be touched. These points are factors that give image a great degree of freedom as well as three-dimensionality. Rakkolainen et al. have constructed an interactive system that applies this characteristic of being able to directly touch the image, and have identified its potential for use in entertainment and the arts [1,2]. Furthermore, recent years have seen the commercialization of the fog screen that can be used in the same way as a touch screen [4], and it is expected that in future they will become a more familiar sight.

However because a feature of the fogscreen is that light rays are more widely scattered due to the effect of Mie scattering users can only view an image from within a small range of distance from the fog and on the opposite side to the projector [5]. 
Because existing systems such as [1,2] have a limited viewable range, the relative positioning of the viewpoint, the fog and the projector are fixed.

Therefore, Yagi et al. have developed a fogscreen that can be viewed from multiple directions by utilizing a cylindrical of screen that allows for Mie scattering and multiple calibrated projectors [5]. Meanwhile, in the Pocket Cosmos developed by Mun et al. [6], the device which generates the fog is fitted with a 3-axis accelerometer, allowing a mobile fogscreen where the fog is directed vertically.

However, although the system developed by Yagi et al. allows freedom in the relative position of the view point and the fog, the relative positions of the fog and the projector remain fixed. And, while the device of Pocket Cosmos [9] has mobility, it doesn't allow for multiple viewpoints, so it is likely that the image on the fog screen can only be viewed by the person holding the device.

As mentioned above, a feature of the fogscreen is its subtle outline, and prior research has suggested its application possibilities in entertainment and the arts, so if a system could be developed which allows for multiple viewpoints and mobility, and also the simultaneous use of multiple devices, it could contribute to the advancement of entertainment and the arts.

Therefore we propose a method to develop a fogscreen that allows multiple viewpoints and mobility, and the simultaneous use of multiple devices. And we carry out an evaluation through the development of "HANASUI", a system based on this method.

HANASUI is system that utilizes a portable, multi-user fogscreen for entertainment. Therefore, we carry out an evaluation through actual user experience to establish whether we have in fact developed a multi-user, portable display, and achieved an eye-catching production.

\section{Proposed Method}

Here we lay out the development of a portable, multi-user fogscreen using the following method. The process we propose flow we propose is illustrated in figure 1.

1. In order to be observable from multiple viewpoints, several projectors are used simultaneously.

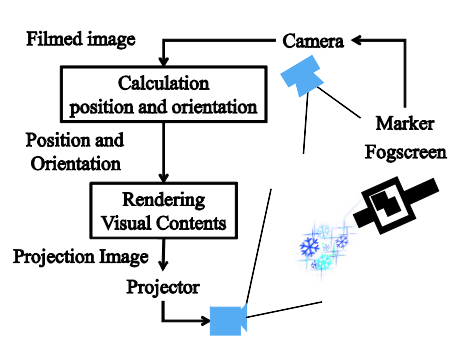

Fig. 1. System Flow

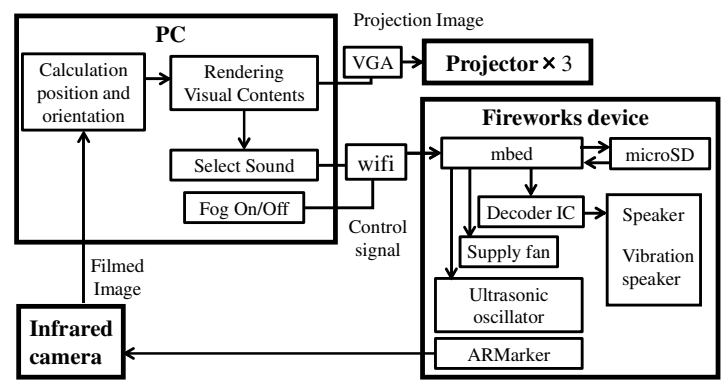

Fig. 2. Construction of HANASUI 
2. In order that the image is correctly projected when the fog generation point is moved, markers were fitted to the fog generator to read its position and orientation and adjust the projection to match those readings.

How to position the projectors when multiple users are using the system simultaneously, it is necessary for a given user to be able to see the images projected onto the other users' fog screens. In order to achieve this, several projectors are arranged. Here, taking into consideration Mie scattering, we positioned three short focus projectors at approximately 60-degree intervals, at the same level and equidistant from the center of the circle, and with their lamps aimed at the same central point. With this arrangement, we avoid images projected from each projector overlapping due to the directionality of scattered light, and it is possible to construct a fogscreen that can be observed from multiple viewpoints.

\subsection{Detecting the Position and Orientation of the Device}

In order to detect the position and the orientation of the device, we use markers of known shape and size and a camera with known internal parameters. If these parameters are known, we can know the position and orientation of the markers [10]. Also, in order to avoid interference with marker recognition when the images are projected onto the markers, an infrared camera and infrared floodlight are used and the markers are filmed in the infrared spectrum.

\subsection{How to Create the Projector Output Images}

In order that each projector can project an image on the fog, it is necessary to create images adjusted according to the projection position of each projector, using the information collected in Subsection 2.2 about the position and orientation of the device. To generate these images, we construct and use a virtual space that is geometrically aligned with the actual space. Geometrical alignment means that the position of the cameras set up to film the markers in the virtual space (virtual marker cameras) and the relative positioning of the projectors (virtual projectors) are aligned with the infrared cameras filming the markers and the projectors in the actual space. By setting up this alignment in advance, the detected marker positions and orientation can be directly reflected in the virtual space.

The acquired the position and the orientation of the device are reflected the device arranged in the virtual space (virtual device) and we arrange the image we want to project on the front of the virtual device. Furthermore, we arrange the virtual cameras in the locations of each of the virtual projectors. We set the field of view of the virtual cameras to be the same as that of the projectors. With this setup, if the virtual cameras film the image at the front of the device, these filmed images are the images are the images that geometrically aligned projectors must project. 


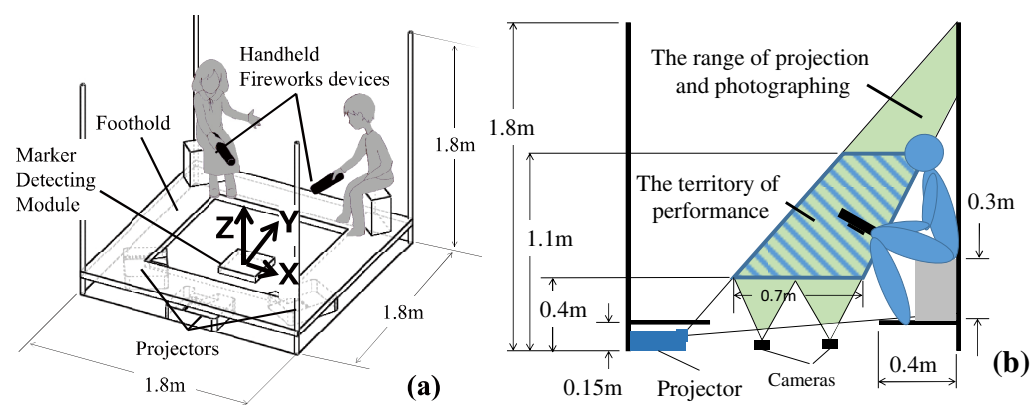

Fig. 3. a) Configuration of HANASUI b) Territory of performance

At the time of this study, the geometrical alignment of the real and virtual spaces was not automated. A hexagonal prism was arranged in both the real and virtual spaces; the prism was filmed by the virtual cameras; these images were projected; and the projectors were manually moved and adjusted until the projected prism aligned with the actual prism.

\section{Implementation of HANASUI}

In the section, we cover the implementation of HANASUI, the system through which we developed our purposed multi-user and portable fogscreen. The configuration of the HANASUI system is illustrated in figure 2, and the system as whole is illustrated in figure 3.

HANASUI is made up of a performance stage and a handheld device (the handheld fireworks device figure 4).

The user experiences the HANASUI by holding out the device above the central area of the performance stage.

\subsection{Performance Stage}

The performance stage is in a darkroom $1.8 \mathrm{~m}$ high and $1.8 \mathrm{~m}$ square, and a platform with a $1.1 \mathrm{~m}$-square hollow center is located approximately $15 \mathrm{~cm}$ above the floor. Below the platform three short focus projectors (Casio XJ-ST155 1024x768) are arranged at approximately 60-degree intervals, facing the center of the hollow. On the floor of the hollow space, facing directly upwards, are an infrared floodlight (WTWF6085) and two infrared cameras (Logicool HD Pro WebCam C910 1920x1080).

In addition, the modularized infrared floodlight and infrared camera is referred to as a marker detection module. 


\subsection{Marker Detection Module}

The marker detection module has the infrared floodlight at the center, and the infrared cameras are placed symmetrically. By using infrared light, it is possible to reliably detect the markers unaffected by light from the projector in the darkroom.

The infrared floodlight and infrared camera are positioned facing vertically upward ceiling means because it is believed that in this way occlusion will not occur when the user peers into the center of the system from above the device. And the use of two cameras is in order to widen the field of recognition.

\subsection{AR Markers}

With the $1.8 \mathrm{~m}$ square size of the performance stage and the efficiency of the projectors used on this occasion, $1.3 \mathrm{~m}$ above the floor was the highest point in the projectors' projection range. Therefore, as a marker that the camera could recognize up to this point, an $80 \mathrm{~mm}$-square AR marker was placed in the center of a $95 \mathrm{~mm}$-square acrylic plate.

To estimate the marker's position and orientation, an ARToolKit was used, which could estimate position and orientation at 30fps.

\subsection{Fireworks Device}

The fireworks device (figure 4) plays audio in time with the fog dispersal and image. The fireworks device is fitted with an ultrasonic oscillator, water storage tank, ventilation fan, mbed (microcontroller), speaker, vibration speaker, wireless module, battery, microSD, MP3 decoder IC, and AR marker, and can be controlled wirelessly.

These components are controlled by the internal microcontroller with the signal sent from a computer. The fog is generated using the ultrasonic oscillator, and about $90 \mathrm{ml}$ of water is spouted as fog per hour. Furthermore, the form of fog screen from user's view is approximately inverted triangle of which height is $0.15 \mathrm{~m}$ and base is $0.15 \mathrm{~m}$. About 10 users can see the contents on the fog simultaneously. The water tank can hold about $40 \mathrm{ml}$ of water so fog can be generated for a maximum of about 20 minutes. Fitting vibration speakers, which emit sounds when it vibrate and are attached to the device, mean that the user experiences vibration simultaneously with audio playback. Ventilation from the fan ensures that the fog doesn't disperse and the projected image is vivid.

\subsection{Contents}

For the purposes of the test, the experience lasts for three minutes. During that time, six visual contents are shown and switched every 30 seconds. Each time, the visual contents are displayed in the order: flames, cherry blossom petals, sunflower, fireworks, Japanese maple leaf, and snowflake. This sequence was first carried out like a regular fireworks display, and subsequently became a conscious representation of the four seasons. 
Each of them is like a performance that is spouted from the front of the handheld fireworks device. Figure 5 shows the view of the fireworks from the user's viewpoint. Also, six varieties of audio were played to match the visual contents. They were played in the following order: the sound of an open fire burning, the song of a Japanese bush warbler and a "shishiodoshi" (a water-filled bamboo tube which clacks

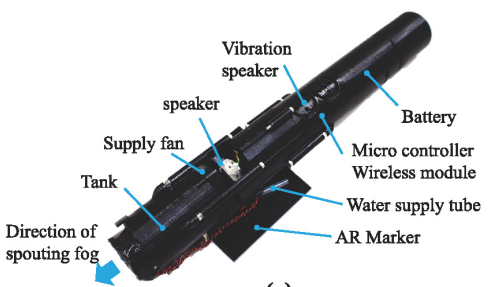

(a)

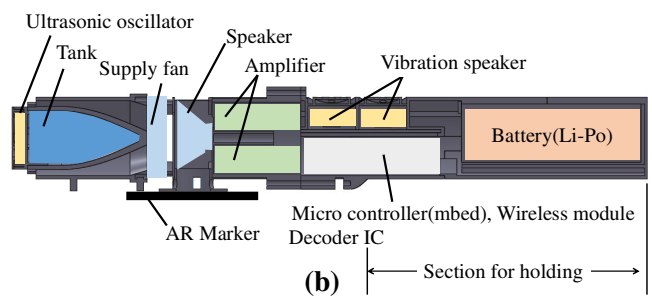

Fig. 4. a) Photograph of fireworks device b) Cross Section of fireworks device
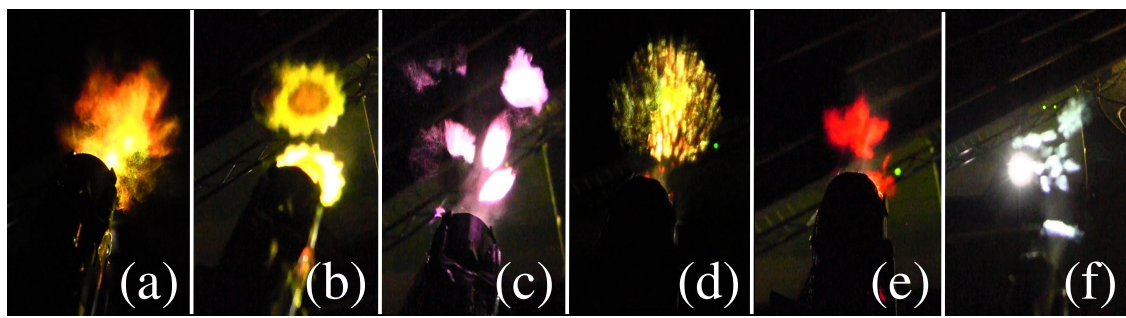

Fig. 5. Virtual contents a) Fire b) Sunflower c) Cherry blossom d) Skyrocket e) Japanese maple f) Snowflake

against a stone when emptied), the chirping of a cicada, the explosion of fireworks, the chirping of a cricket, and the tinkling of a bell.

\section{Evaluation Experiment}

The evaluation experiment was exhibited on October 24-26, 2013 at the IVRC [8] (International Collegiate Virtual Reality Contest) 2013 Tokyo Finals held at the National Museum of Emerging Science and Innovation.

\subsection{Evaluation Experiment}

In order to evaluate the system, users were given a questionnaire. 162 questionnaires were collected from visitors aged between 7 and 68 years old.

The questionnaire consisted of six questions to which respondents awarded scores between 1 and 5, and a free description. The questions are shown in table 1, while the scoring scales by Likert scale from 5(yes) to 1(no). The results for question 5 are based on 145 questionnaires, and relate to the experience of two users each holding a 
fireworks device. For this question, any cases where a user did the experiment alone were marked as a non-respondent.

Table 1. The questions of questionnaire

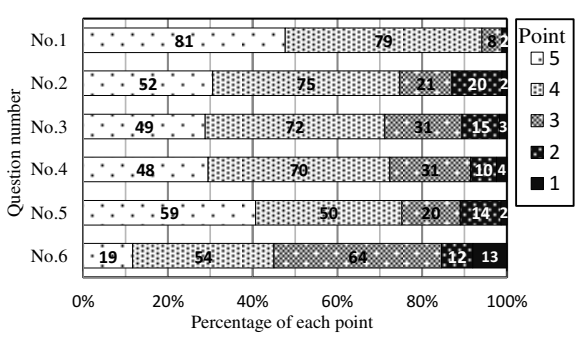

\begin{tabular}{|c|l|}
\hline & \multicolumn{1}{|c|}{ Question items } \\
\hline No.1 & Do you think that the performance of HANASUI is beautiful, or not? \\
\hline No.2 & $\begin{array}{l}\text { Could you look like the projected contents is emitting from the top of } \\
\text { fireworks device, or not? }\end{array}$ \\
\hline No.3 & $\begin{array}{l}\text { Did you think the projected contents on fogscreen was tracking } \\
\text { fireworks device, or not? }\end{array}$ \\
\hline No.4 & Did the sound of fireworks device make NAHASUI more attractive? \\
\hline No.5 & Could you watch the virtual contents projected on fogscreen, or not? \\
\hline No.6 & Did the vibration of fireworks make HANASUI more attractive? \\
\hline
\end{tabular}

Fig. 6. Results of Questionnaire

The procedure was that before the experience, the details of the system were explained as, "HANASUI is a performance system that uses fog and projectors in a handheld firework-like device," along with how to hold the device and the fact that the usable range of the fog screen is above the hollow central part of the performance stage. Questionnaire answers were requested after the experience.

\subsection{Results}

The results of the collected questionnaires are shown in figure 6. It shows the proportional breakdown for each score, with the width of the graph corresponding to $100 \%$.

Free Description. In relation to the evaluation questions, there were opinions related to inadequate sound or vibration, such as, "the sound should be louder," or "I couldn't feel the vibration." But on the other hand, there were comments such as, "the sound was very good," and "the vibration was wonderful."

With regard to users' impressions, in addition to the comment, "it was beautiful," there were many comments saying, "it was magical."

Other than that, many users pointed out issues related to the particular positional setup of the fog screen, such as "when the projector light got in my eyes it was blinding."

Observations. From question 1, more than $90 \%$ of users felt the HANASUI performance was beautiful. And with many comments saying it was magical, we can say that it is established as a form of performance.

From question 2, we can say that many users felt that the visual contents seemed to be spouted out from the front of the device.

The results of question 3 tell us that many users felt like the visual contents followed the front of the handheld fireworks device.

From the results of questions 2 and 3, we can say that we have succeeded in developing a mobile fog screen. 
Regarding sound and vibration, although the majority of users found that they added to the appeal of the performance, many others did not feel that way. It is likely that the sound volume was inadequate, and so was influenced by sound from the surroundings.

From question 5, we know that many users were able to view the image projected on the other user's fog. On this occasion, no evaluation was carried out of the precision of the projected visual contents, but more than $75 \%$ of users gave an evaluation that was "just about make it out" or better. So we can see that we have succeeded in developing a multi-viewpoint fog screen.

\section{Conclusion}

Here, we have proposed method to develop a multi-viewpoint and portable fog screen using AR markers and virtual space, and we have produced the HANASUI system using that method. And we carried out an evaluation experiment using a questionnaire.

From the results, we have confirmed that many users were able to view the visualcontents projected on another user's fog screen, and that they felt the visual contents were in front of the fog screen-generating device they themselves were holding.

From the above points, we have confirmed that the method proposed here for constructing a multi-viewpoint and portable fog screen is viable. We have also confirmed that many users find the HANASUI system developed using this method "beautiful" and "magical."

HANASUI was awarded the Maywa Denki Presidents Prize at IVRC 2013. Video of the experience and from the user's viewpoint can be seen on YouTube [9].

\section{References}

1. Rakkolainen, I., Palovuori, K.: Walk-thru screen. In: Electronic Imaging International Society for Optics and Photonics, pp. 17-22 (2002)

2. Rakkolainen, I.: Tracking users through a projection screen. In: Proceedings of the 14th Annual ACM International Conference on Multimedia, pp. 101-104 (2006)

3. Hayashi, H., Onishi, R., Hirai, S.: Spacial Visual Expression Using Mist in A General Bathroom-Interactibity with Touch Sensor Embedded in A Bathtub. In: EC 2007, pp. 7576 (2007) (in Japanese)

4. DISPLAIR play with air, http: / / displair.com/ (accessed December 7, 2013)

5. Yagi, A., Imura, M., Kuroda, Y., Osiro, O.: Multi-Viewpoint Interactive Fog Display. TVRSJ 17(4), 409-417 (2012) (in Japanese)

6. Mun, N., Sone, J., Natsui, N., Hasebe, T., Yoshida, K.: Pocket cosmos-Cosmos in my hand. The Jornal of the Society for Art and Science 3(4), 244-249 (2004) (in Japanese)

7. Kato, H., Billinghurst, M.: Marker tracking and hmd calibration for a video-based augmented reality conferencing system; In Augmented Reality. In: Proceedings of the 2nd IEEE and ACM International Workshop on IEEE, pp. 85-94 (1999)

8. IVRC 2013 official home page, http: / / ivrc. net/2013 / (accessed July 10, 2014)

9. Hanasui, http://www.youtube.com/watch?v=_kLVEq-qVu4 (accessed December 8, 2013) 